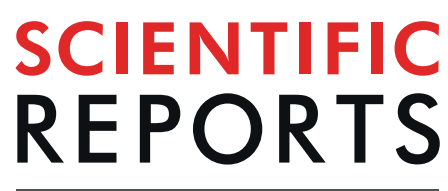

natureresearch

\title{
One-step artificial antigen presenting cell-based vaccines induce potent effector CD8 T cell responses
}

\author{
Qingtai Su $(1)$ \& Botond Z. Igyártó ${ }^{*}$ \\ The production and wide use of artificial antigen presenting cells (aAPCs) in the clinic as cancer \\ immunotherapeutics are hindered by the need of identifying immunogenic cancer antigens and \\ production of recombinant patient-specific major histocompatibility complexes (MHC) loaded with \\ these peptides. To overcome these limitations, in this study, we tested the idea of whether peptide- \\ MHCs can directly be captured from cell lysates, including cancer cells using affinity beads, and used \\ to initiate T cell responses. In theory, these affinity beads covered with the unknown peptide-MHC \\ repertoire captured from the cancer cells could interact with a wide range of antigen-specific $T$ cells and \\ promote anti-cancer responses. Indeed, we found that we can successfully pull-down peptide-MHCs \\ from cell lysates and the aAPCs generated using this technique were able to induce antigen-specific \\ cytotoxic effector $\mathrm{T}$ cell responses that led to in vitro and in vivo tumor cell killing. In summary, we \\ present here a novel technique to generate patient-specific aAPCs, that might have the potential to \\ revolutionize the field of cancer vaccines, and provide patients with a vaccine in matters of days at \\ minimal costs.
}

Dendritic cells (DCs) as professional antigen presenting cells (APCs), coordinate every aspect of immunity ${ }^{1}$. DCs stimulate and train other immune cells, such as T cells, of the adaptive immune system by presenting peptide epitopes derived from antigens (self, cancer neoantigens, foreign, etc.) on their MHC, and providing co-stimulatory signals in membrane bound and secreted (cytokines) forms $\mathrm{s}^{1,2}$. These three signals train the $\mathrm{T}$ cells to recognize, destroy or tolerate the cells that carry these antigens. Thus, not surprisingly the DCs are the main target of immunotherapies. Two main strategies are used to exploit DCs for cancer therapy ${ }^{3}$. One of them require isolation of patient monocytes from blood and complex in vitro manipulation that involves differentiation and maturation into DCs using cytokine and adjuvant cocktails and pulsing with the chosen antigen(s)/cell lysates, followed by reinfusion into the patient. An alternative approach uses antibody as a carrier to deliver antigens to DCs in vivo. However, both of these approaches showed only limited success rate, mainly because the DCs' function is highly dependent on the tumor environment which is often time inhibitory ${ }^{2,3}$.

Artificial antigen presenting cells (aAPCs) could be a viable alternative to live DCs since they do not react to the environmental clues ${ }^{4-11}$. However, present day aAPCs are limited by the fact that researcher conjugate known, genetically engineered and expressed peptide-MHC on beads to stimulate T cell responses - a method limited to defined and selected MHC/peptide complexes. The need of prior identification of immunogenic tumor neoantigens and in vitro synthetization of corresponding patient-specific MHC-I haplotype has limited the widespread use of aAPCs in the clinic ${ }^{12,13}$.

Here we present an alternative approach to generate aAPC-based cancer vaccines that does not require identification and in vitro production of the peptide-MHCs. This is a one-step process that allows the capture of the peptide-MHCs directly from the patient-derived tumor cell lysates to generate aAPCs. We bring experimental evidence that peptide-MHC-I repertoire of normal- or tumor cells can be successfully captured directly from cell lysate using affinity beads. The aAPCs generated using this technique were able to induce antigen-specific cytotoxic effector $\mathrm{T}$ cell responses that led to in vitro and in vivo tumor cell killing. Collectively, our novel aAPCs production strategy show potential in revolutionizing aAPC-based cancer immunotherapy. 


\section{Materials and Methods}

Mice. OT-I Rag2-1- CD8 TCR transgenic mice specific for OVA $257-264$ (B6.129S6-Rag2tm1Fwa $\mathrm{Tg}(\mathrm{TcraTcrb}) 1100 \mathrm{Mjb})$ presented on $\mathrm{H}-2 \mathrm{~Kb}$ and $\mathrm{WT} \mathrm{C} 57 \mathrm{BL} / 6$ mice were purchased from Taconic Biosciences (Rensselaer, NY). All experiments were performed with 8 to 26-week-old female and male mice. Mice were housed in microisolator cages and fed autoclaved food and acidified water. The Baylor Institutional Care and Use Committee approved all mouse protocols. All experiments were performed in accordance with relevant guidelines and regulations.

Cell lines. B16-OVA (B16F10 tOVA GFP, expressing truncated OVA and GFP) and parental B16F10 are a gift of Drs. Michael Gerner and Andrew Oberst (University of Washington). HEK293T cell line was purchased from ATCC (Manassas, VA). Cells were cultured in Dulbecco's Modified Eagle Medium (Gibco, Grand Island, NY) supplemented with $10 \%$ FBS, $1 \%$ Glutamax and $1 \%$ sodium pyruvate.

$\mathrm{H}-2 \mathrm{~Kb} / \mathrm{OVA}$ expression. The $\mathrm{H}-2 \mathrm{~Kb}$ sequence was sub-cloned into cetHS-puro plasmid. As a result, Ctag sequence was fused to the $\mathrm{C}$-terminus of the $\mathrm{H}-2 \mathrm{~Kb}$ sequence. The successful generation of the construct was determined by PCR and sequencing (data not shown). One day prior to transfection the HEK293T cells were seeded in $10 \mathrm{~cm}$ tissue culture dish. By next day the cells reached $70-80 \%$ confluence. At this time, the culture medium was replaced with $9 \mathrm{~mL}$ DMEM medium containing $25 \mu \mathrm{M}$ chloroquine and the cells transfected with plasmids coding for Kb-Ctag and OVA (pcDNA3-OVA; Addgene, plasmid \#64599). Briefly, $5 \mu \mathrm{g}$ of Kb-Ctag and $5 \mu \mathrm{g}$ OVA expressing plasmids were mixed in $450 \mu \mathrm{L} \mathrm{H}_{2} \mathrm{O}$ in $1.5 \mathrm{~mL}$ Eppendorf tube; $500 \mu \mathrm{L} 2 \mathrm{X}$ HBSS was added sequentially. $50 \mu \mathrm{L} 2 \mathrm{M} \mathrm{CaCl}_{2}$ solution was then added and the tube was vortexed and kept on ice for 15 minutes. The plasmids were gently added on top of the cell cultures. For single transfections $10 \mu \mathrm{g}$ of $\mathrm{Kb}$-Ctag plasmid was used. On day 2 post transfection the cells were washed with warm DMEM medium twice and cultured for one extra day.

aAPC production. Kb-Ctag and OVA expressing $293 \mathrm{~T}$ cells (or Kb-Ctag expressing B16F10 cells) were lysed in lysis buffer (1\%CHAPS, $25 \mathrm{mM}$ Tris $\mathrm{pH} 7.5,150 \mathrm{mM} \mathrm{NaCl})$ containing protease inhibitor (cOmplete ULTRA $^{\text {TM }}$ Tablets; Roche, Mannheim, Germany). Lysis was performed at $4{ }^{\circ} \mathrm{C}$ for 1 hour. Supernatant was acquired by centrifuging the lysate at $12,000 \mathrm{rpm}$ for 20 minutes. The cleared lysate was then mixed with Ctag matrix (CaptureSelect ${ }^{\mathrm{TM}} \mathrm{C}$-tag Affinity Matrix, Thermo Scientific, Waltham, MA) and incubated at $4{ }^{\circ} \mathrm{C}$, on a slowly rotating surface for one hour. The matrix was then washed extensively with sterile PBS ( $500 \mathrm{rpm} / 20 \mathrm{sec}$ onds spin was used to recover the matrix). The successful pull-down of Kb:SIINFEKL pMHCI complex (or Kb) was determined by staining the matrix with antibodies that detect $\mathrm{Kb}$ and/or SIINFEKL bound to $\mathrm{H}-2 \mathrm{~Kb}$.

In vitro OT-IT cell activation. Secondary lymphoid organs from OT-I Rag1 ${ }^{-1-}$ mice were smashed through cell strainers and the red blood cells lysed using ACK. After washing, the cells were used as is or labeled with cell trace violet (CellTrace ${ }^{\mathrm{TM}}$ Violet Cell Proliferation Kit, Invitrogen, Carlsbad, CA) according to the manufacturer's instructions. The cells were then seeded in a 24 -well plate in complete RPMI medium, each well containing 4 million cells in $2 \mathrm{~mL}$ medium. Control and experimental aAPCs $(20 \mu \mathrm{L}$ matrix for one well) were added to the cell cultures for 6 days. Every other day, half of the medium was replaced with fresh medium.

Flow cytometry. Staining was performed as previously described ${ }^{14}$. Intracellular cytokine staining was performed with the BD Bioscience Cytofix/Cytoperm kit (BD Biosciences, San Jose, CA), according to the manufacturer's instructions. Samples were analyzed on LSRFortessa flow cytometer (BD Biosciences, San Jose, CA). The fluorochrome-conjugated antibodies to IFN gamma (XMG1.2), granzyme B (QA16A02), H-2Kb bound to SIINFEKL (25-D1.16), CD3ع (145-2C11), CD44 (IM7), CD90.1 (OX-7) and CD8a (3-6.7) were purchased from BioLegend (San Diego, CA). Anti-Kb antibody (Y-3) was purchased from BioXCell (West Lebanon, NH) and conjugated with Alexa Fluor ${ }^{\mathrm{TM}} 647$ antibody labeling Kit (Invitrogen, Carlsbad, CA). Data were analyzed with FlowJo software (TreeStar; Ashland, OR). All the flow cytometric plots displaying cells were pre-gated on live cells using Fixable Viability Dye eFluor 780 (eBioscience, San Diego, CA) and singlet events.

Adoptive transfer of OT-IT cells into tumor bearing mice. Eight-to-twelve weeks old female and male WT C57BL/6 mice were inoculated subcutaneously with $10^{6} \mathrm{~B} 16$-OVA cells. When the tumors became palpable (day 7) each mouse received through tail vein injection $4 \times 10^{6}$ OT-I T cells stimulated in vitro with SIINFEKL, control or Kb/OVA aAPCs for 5 days. Female OT-I mice were used as T cell source. Tumors were measured using a caliper at the indicated time points and tumor volumes calculated based on the following formula: volume $=\left(\mathrm{W}^{2} * \mathrm{~L}\right) / 2$, where $\mathrm{W}$ is width and $\mathrm{L}$ is length. As per approved animal protocol, the mice in which the tumor size has reached $1,000 \mathrm{~mm}^{3}$ or the animals showed distress such as visible weight loss, lack of grooming and feeding were euthanized.

Statistical analysis. Statistical analyses were performed using GraphPad Prism7.0 software (Graphpad, La Jolla, CA). Statistical methods used to determine significance are listed in the figure legends. A p value $<0.05$ was considered statistically significant.

\section{Results}

The concept behind one-step aAPCs. The production and wide use of aAPCs as cancer immunotherapeutics is hindered by the need of identifying immunogenic cancer antigens and production of recombinant patient-specific HLAs loaded with these peptides. To overcome these limitations, we tested the idea whether peptide-MHCs can directly be captured from cell lysates, including cancer cells using affinity beads. In theory, these affinity beads covered with the unknown peptide-MHC repertoire captured from cancer cells could interact 


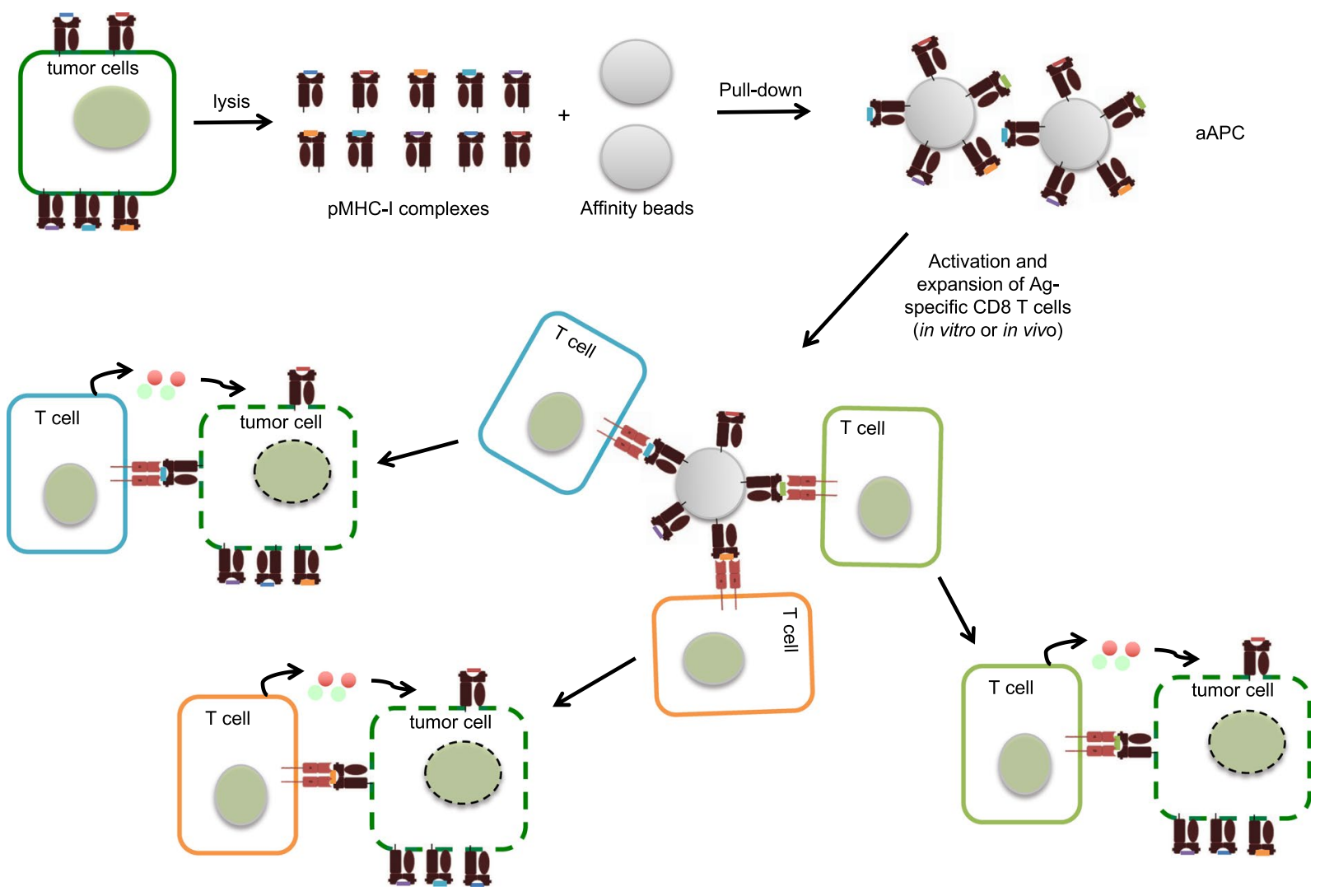

Figure 1. Graphical abstract. Graphical representation of one-step aAPC generation. Tumor cell lysates are incubated with affinity beads that capture the peptide-MHC-I repertoire of the cancer cells. The beads are then used to activate cancer antigen-specific T cell clones that will ultimately kill the tumor cells.

with a wide range of antigen-specific T cells and promote anti-cancer responses. The concept and strategy to generate one-step aAPCs are depicted in Fig. 1/Graphical abstract. For the proof of concept experiment we designed a system where we can control and confirm each step of the aAPC generation. As a first step we transfected human embryonic kidney cells (HEK293T) with plasmids coding for C-tagged mouse H-2Kb (MHC-I) and OVA (Fig. 2a). The expression of the mouse $\mathrm{H}-2 \mathrm{~Kb}$ on the cells surface was detected by Y-3 antibody (recognizes $\mathrm{H}-2 \mathrm{~Kb}$ ) and the formation of OVA-derived peptide MHC complexes with the use of antibody that recognizes SIINFEKL (dominant, OVA-derived CD8 epitope) bound to $\mathrm{H}-2 \mathrm{~Kb}$. We observed efficient expression of $\mathrm{H}-2 \mathrm{~Kb}$ and loading of SIINFEKL peptide on MHC-I molecules (data not shown). No SIINFEKL staining was observed in the cells transfected only with Kb-Ctag plasmid (data not shown). After confirming the expression of OVAderived peptide in the context of MHC-I, we lysed the cells and purified the peptide-MHC-Is using affinity matrix targeting the C-tag motif of the H-2Kb. The capture of MHC-I and MHC-I complexed with SIINFEKL were determined with the use of antibodies already presented above. We found that the affinity beads efficiently captured MHC-I and MHC-I loaded with SIINFEKL (Fig. 2a). Thus, these data suggested that affinity beads targeting MHC-I could be an easy way to generate patient-specific aAPCs.

aAPCs loaded with Kb:SIINFEKL can prime OT-IT cells. The successful generation of aAPCs using affinity matrix prompted us to test whether the generated aAPCs can activate antigen-specific CD8 T cells. For this purpose, we generated aAPCs as presented above and co-cultured them with naïve OT-I cells. OT-I cells recognize SIINFEKL peptides presented in the context of $\mathrm{H}-2 \mathrm{~Kb}$. SIINFEKL peptide $(2 \mu \mathrm{g} / \mathrm{mL})$ stimulation served as a positive control (Pulle et al., 2006). The ex vivo culturing of OT-I T cells with aAPCs carrying Kb:SIINFEKL (Kb/OVA), but not control aAPCs (Kb), led to cell cluster formation (Fig. 2b). Inclusion of Y-3 antibody in cultures that binds to peptide groove of the $\mathrm{H}-2 \mathrm{~Kb}$, and thus interfere with TCR binding, decreased OT-I cluster formation (Fig. 2b). To confirm the activation and proliferation of OT-I cells, CTV-labeled OT-I cells were co-cultured with either control or Kb/OVA aAPCs and analyzed by flow cytometer. Kb/OVA aAPCs, unlike control aAPC, induced significant upregulation of CD44 and dilution of the proliferation dye by day 2 (Fig. 2c). AAPCs generated using the same technique but a different cell line (B16F10) provided similar results (data not shown). Together, these data suggest that our aAPCs were able to prime antigen-specific T cell responses.

aAPC-activated T cells can kill tumor cells in vitro and in vivo. To investigate the cytotoxic potency of the aAPC-primed T cells, we designed an in vitro tumor cell-killing assay using the B16F10 cell line. OT-I T cells were first primed by Kb/OVA aAPCs or control aAPCs for 6 days in vitro (Fig. 3a). Prior use the cytotoxic phenotype (IFN $\gamma$ and granzyme B) of the primed OT-I cells were confirmed by flow cytometry (Fig. 3b). B16F10 
a).
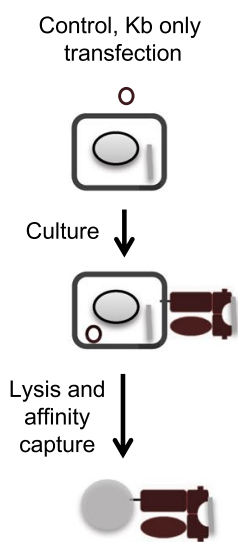

Control aAPC

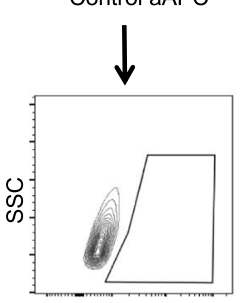

Kb:SIINFEKL

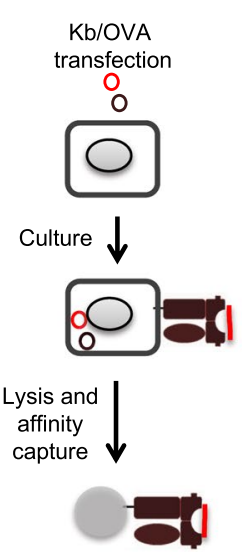

$\mathrm{Kb} / \mathrm{OVA}$ aAPC

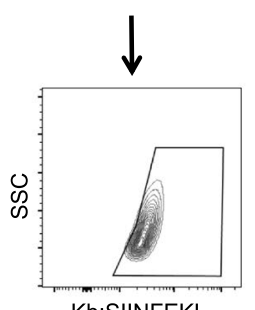

b).

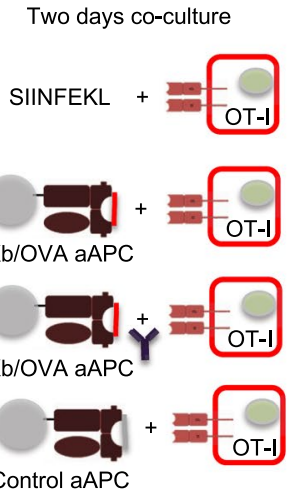

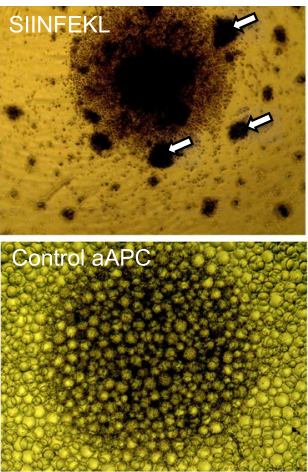

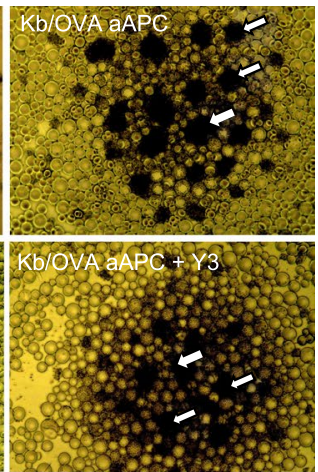

c).

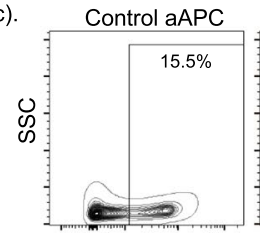

CD44

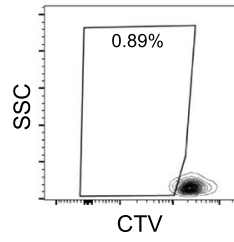

$\mathrm{Kb} / \mathrm{OVA}$ aAPC

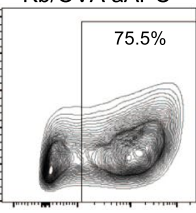

CD44
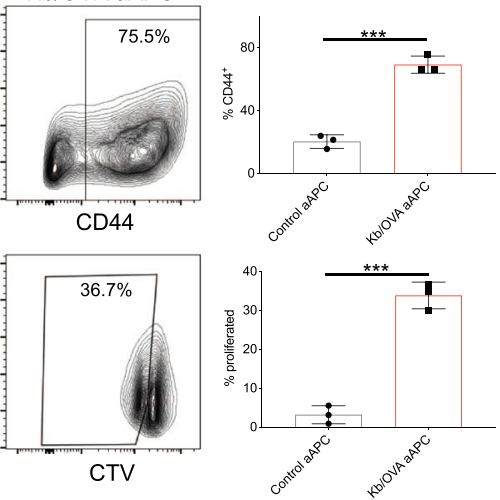

Figure 2. aAPCs loaded with Kb:SIINFEKL can prime OT-I T cells. (a) Experimental flow: 293T cells were transfected with either Kb-Ctag (brown) coding plasmid alone or in combination with OVA (red) coding plasmid. After two days of culture the cells were lysed and the MHC-Is captured using affinity beads targeting the Ctag motif. The capture of MHC-I complexed with SIINFEKL were determined with the help of flow cytometry using antibody that recognizes SIINFEKL bound to Kb. One representative experiment out of three is shown. (b) OT-I cells were stimulated in vitro with SIINFEKL peptide, Kb/OVA aAPCs, control aAPCs or Kb/ OVA aAPCs in the presence of Y3 antibody, and pictures taken two days later (right). Arrows point to some of the activated OT-I cell clusters induced by the stimulation. One representative experiment out of three is shown. Three samples/group, except SIINFEKL that served the role of positive control. (c) In a separate experiment, OT-I cells were stimulated with either Kb/OVA aAPCs or control aAPCs for 2 days and assessed by flow cytometer for activation (CD44) and proliferation (CTV dilution). One representative experiment out of three is shown, $\mathrm{n}=3$. Unpaired t-test. $* * * \mathrm{p}<0.001$.

cells that express OVA and GFP (hereafter B16-OVA) were then mixed with their parental B16F10 cells (B16 WT) that were labeled with CTV at a ratio of 1:1 (Fig. 3a). The primed OT-I T cells were added directly to B16 cell cultures one day after seeding at an effector to tumor cell ratio of 2:1. Peptide-stimulated OT-I cells served as positive controls. Cell counts were read by FACS one day after T cell addition. Both peptide- and aAPC-stimulated T cells effectively killed the tumor cells expressing OVA, but left the parental WT tumor cells intact (Fig. 3c). To further interrogate the cytotoxic ability of aAPC-stimulated T cells, we infused the OT-I T cells intravenously into WT mice carrying B16-OVA tumors (Fig. 4a). Both peptide and aAPC-stimulated OT-I cells significantly slowed the tumor growth (Fig. 4b) and increased survival (Fig. 4c). Collectively, our data suggested that this novel aAPC was able to induce cytotoxic T cells and that could potentially be used as immunotherapeutic.

aAPCs generated using tumor cells were able to activate $T$ cells from tumor-bearing mice. The success with the model antigen OVA, encouraged us to explore whether aAPCs generated by using unknown tumor antigens could be used to stimulate $\mathrm{T}$ cells isolated from tumor-bearing mice. To address this question, we first transfected $\mathrm{B} 16 \mathrm{~F} 10$ cells with $\mathrm{C}$-tagged $\mathrm{H}-2 \mathrm{~Kb}$ and isolated the peptide-MHC-Is as presented above. The scientific rational behind this experiment was that these MHC-I molecules will be loaded with tumor antigens and we can then capture the peptide-MHC-I repertoire of the B16F10 cells using affinity beads (Fig. 5a). FACS assay confirmed the successful capture of $\mathrm{H}-2 \mathrm{~Kb}$ from $\mathrm{C}$-tagged $\mathrm{H}-2 \mathrm{~Kb}$ transfected B16F10 cells (Fig. 5b). As next, splenocytes isolated from B16F10 tumor-bearing mice were isolated and stimulated with experimental and control aAPCs. We observed that our experimental aAPCs, comparing to control aAPCs activated significantly higher numbers of CD8 T cells to produce IFN $\gamma$ (Fig. $5 \mathrm{c}$ ). Thus, the data support that generation of patient-specific aAPCs is plausible. 
a).

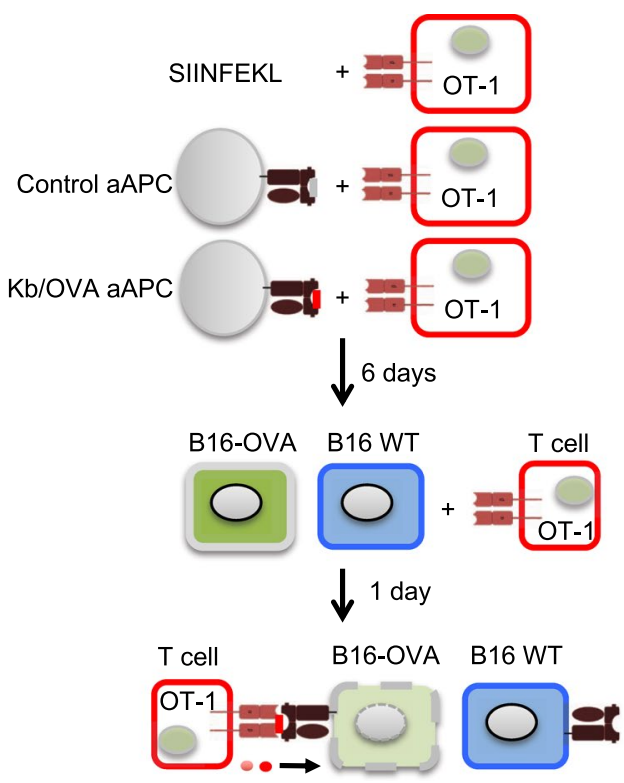

b).
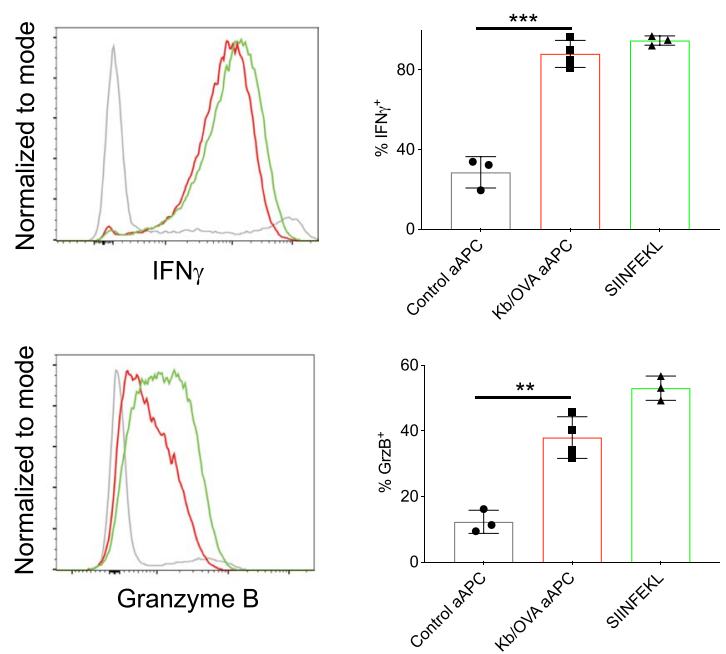

c).
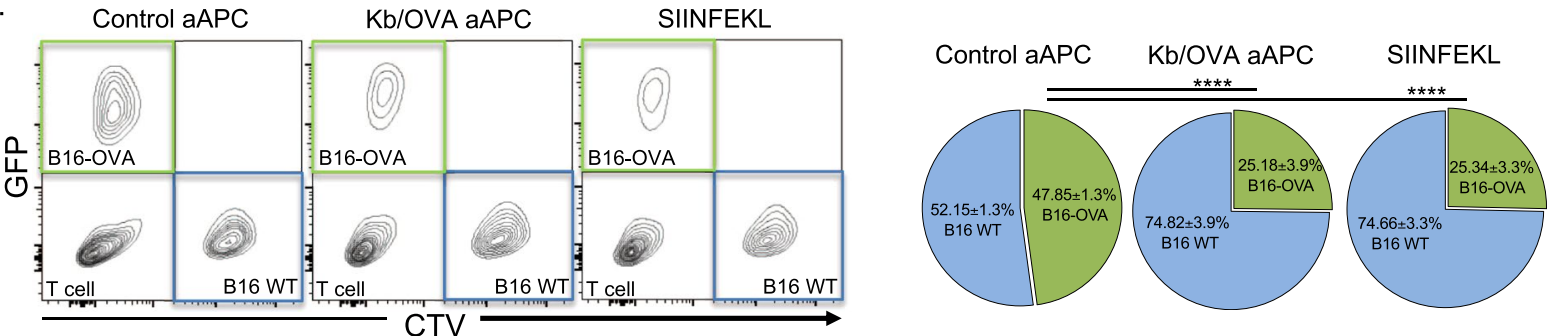

Figure 3. aAPC-activated OT-I T cells can kill tumor cells in vitro. (a) Experimental flow. OT-I cells were stimulated with SIINFEKL, control or Kb/OVA aAPCs for 6 days. (b) Before their use for experiments the OT-I T cells' cytotoxic phenotype was confirmed by flow cytometry. Unpaired t-test, $* * \mathrm{p}<0.01, * * * \mathrm{p}<0.001$. (c) The OT-I cells were then mixed with B16-OVA (target cells; green) and B16 WT (control cells; blue) cells and cultured for one day. The killing of target cells was determined by flow cytometer (left) and shown as relative percentage (right). One representative experiment out of two is shown, $n=3$. One-way ANOVA on green pie segments, $* * * * \mathrm{p}<0.0001$.

\section{Discussion}

Here we present a novel and simple technique to generate patient-specific cancer vaccines. We bring experimental evidence that can serve as the basis of the generation of patient-specific vaccines targeting cancer and autoimmune diseases. We show that affinity beads can be used to pull down peptide-MHC directly from cell lysates and it is a viable option to generate patient-specific aAPCs in matters of days at minimal costs.

The clinical usability of aAPCs generated until now to treat cancer were limited by multiple factors. AAPCs have to be patients specific that first require characterization of the patient's HLA haplotype and identification of cancer neoantigens. The HLA haplotype is fairly easily determined by PCR and flow cytometry, however identification of immunogenic cancer neoantigens is a very expensive, tedious, and labor-expensive process with many caveats along the pipeline that further limit its success rate ${ }^{15-18}$. Since most of the cancers are highly heterogeneous a dominant neoantigen might only target some of the cancer cells, and the neoantigen could be patient-specific or it will only bind to certain HLA molecules, limiting their wider usability. To make the aAPCs, the correct HLAs have to be produced as recombinant proteins and assembled with the corresponding neoantigenic peptides. The recombinant HLA and peptides might not carry all the posttranslational modifications that would normally occur in vivo that could result in less effective TCR stimulation. Furthermore, the whole spectrum of HLA is hard to reconstruct. To simplify the generation of aAPCs, we decided to focus on things that are important and disregard the need to identify antigens. We hypothesized that we could generate aAPCs by simply pulling down peptide-MHCs directly from tumor lysates. Indeed, we found that we can successfully pull-down peptide-MHCs that can later be used to stimulate antigen-specific anti-cancer effector T cell responses. Tumor-specific T cell clones are of low abundance in nature ${ }^{19}$, and unlike most of the aAPCs generated to date, which often only present one antigenic peptide, this technique, by capturing a diverse peptide-MHC-I pool should increase the chance to target and activate multiple cancer-specific $\mathrm{T}$ cell clones. It is expected that the peptide repertoire presented by the cancer cells and the tumor heterogeneity will be represented proportionally on the aAPCs. If later studies will prove it otherwise, we can use a modified version of the Drop-sequencing technology, where in this case a lipid 
a).

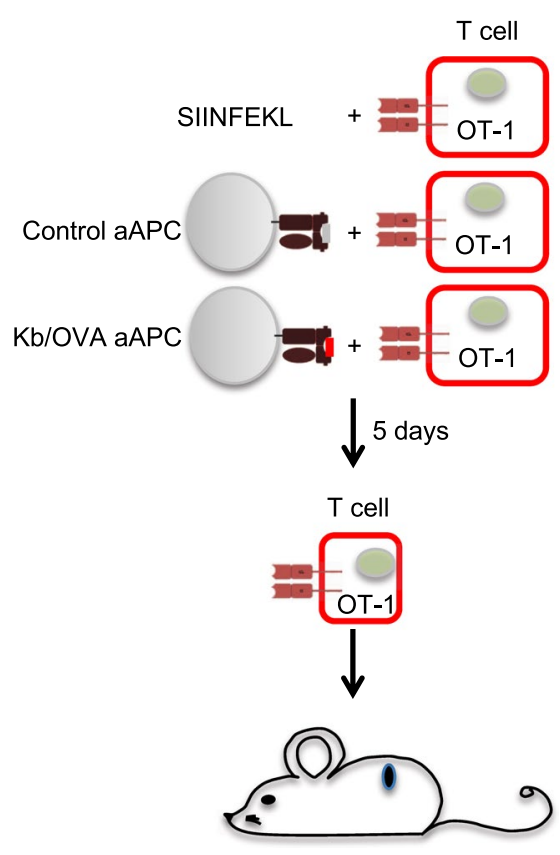

b).

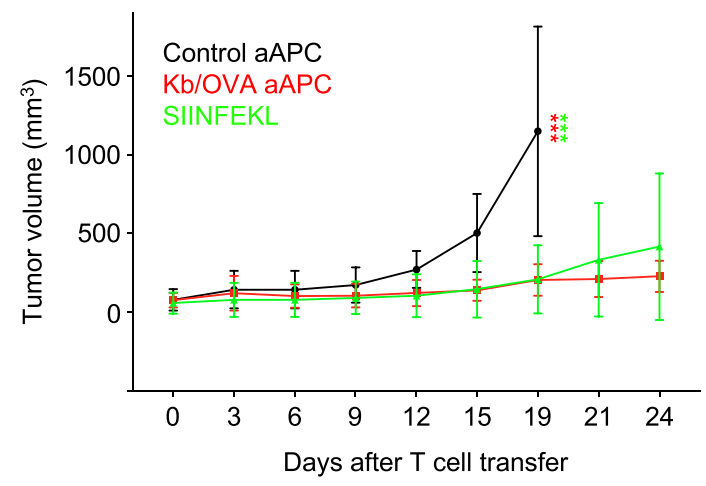

c).

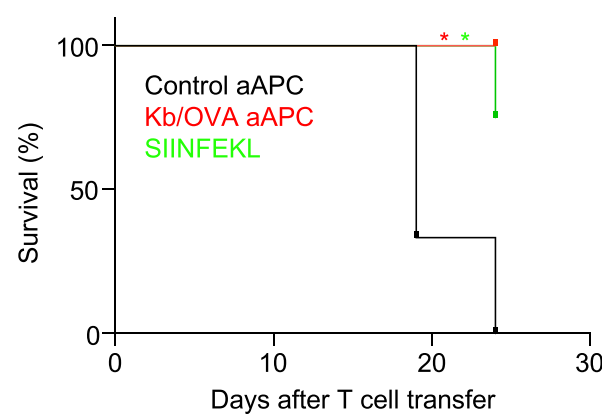

Figure 4. aAPC-activated OT-I T cells can kill tumor cells in vivo. (a) Experimental flow. OT-I cells were stimulated with SIINFEKL, control or Kb/OVA aAPCs for 5 days. To determine the in vivo tumor killing potency of the aAPC-stimulated OT-I cells, the OT-I cells were transferred into B16-OVA tumor bearing mice and the tumor growth $(\mathbf{b})$ (two-way ANOVA; Tukey's test $* * * \mathrm{p}<0.001)$ and the survival monitored as depicted (c) (Long-rank, Mantel-Cox test, $* \mathrm{p}<0.05)$. Data from two experiments were combined. $\mathrm{N}=6-8$ mice/group.

a).

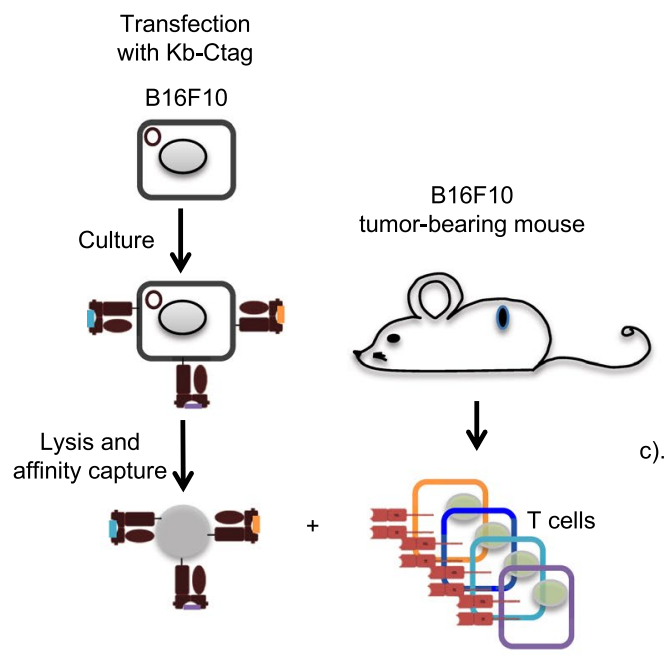

b).

c).
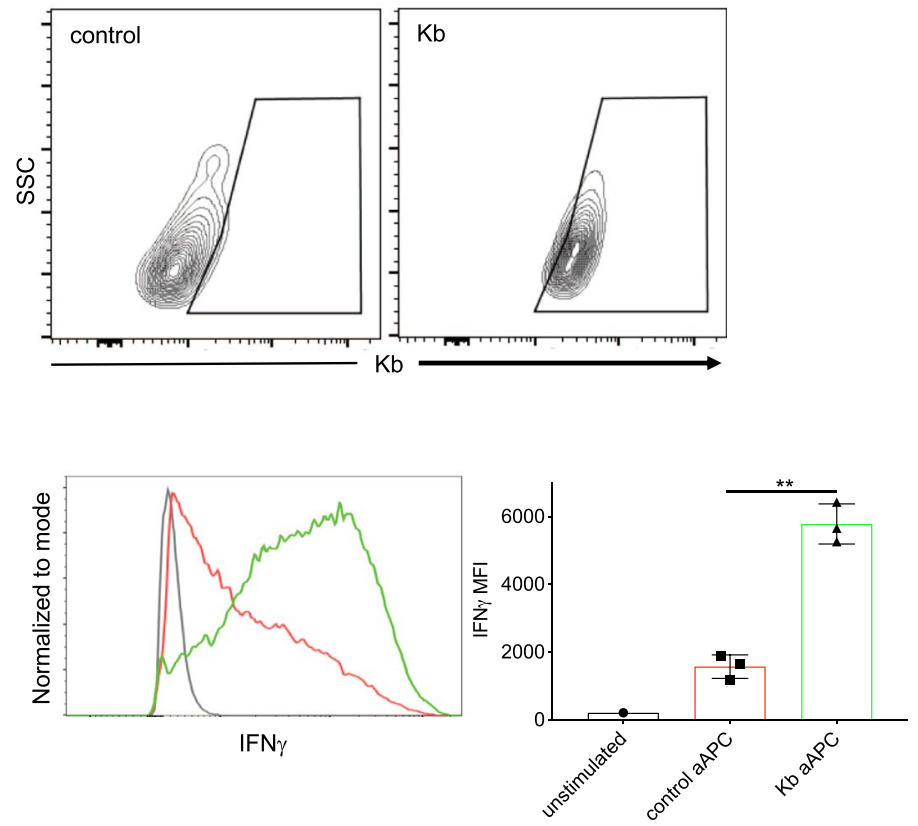

Figure 5. aAPCs generated using B16F10 cells activated T cells from tumor-bearing mice. (a) Experimental flow for data presented on figures $(\mathbf{b}, \mathbf{c})$. B16F10 cells were transfected with $\mathrm{Kb}$-Ctag plasmid. Two days later the cells were lysed and the MHC-I repertoire captured using affinity beads. The successful capture of the MHC-Is was confirmed with flow cytometry $(\mathbf{b})$. The aAPCs were then used to stimulate T cells isolated from tumor bearing mice's spleen for six days. The induction of CD8 T cells with cytotoxic phenotype, characterized by IFN $\gamma$ production, was confirmed using flow cytometry (c). One representative experiment out of two is shown, $\mathrm{n}=3$, except unstimulated $\mathrm{n}=1$. Unpaired t-test. $* * \mathrm{p}<0.01$. 
droplet containing lysis buffer will form around one cancer cell and one affinity bead. This will assure that every aAPC will represent one cancer cell's peptide-MHC-I repertoire. Tumor samples with higher leukocytic infiltrates might require purification steps to enrich for tumor-derived signatures. In some instances tumor cells escape immune surveillance by downregulating surface expression of $\mathrm{MHC}-\mathrm{I}^{20}$. In theory, our aAPCs could still capture the intracellularly retained peptide-MHC-I repertoire; or as a last resort, ectopic transfection of tumor cells with patient-specific HLA-I could circumvent this caveat. The aAPCs generated using this technique will probably contain, self, non-mutated, non-immunogenic peptides that could trigger some sorts of autoimmune responses if combined with co-stimulation. This, however, is expected to be minimal, because of central and peripheral tolerance. The possible autoimmune symptoms could also be controlled by the use of different drugs.

Because of lack of access to antibodies that would recognize the cytoplasmic part of mouse $\mathrm{H}-2 \mathrm{~Kb}$ and for versatility reasons, in these proof-of-concept in vitro and in vivo experiments, we used tag-specific antibodies. Future studies should consider antibodies that recognize cytoplasmic portion of the $\mathrm{Kb}$ or target beta-2 microglobulin to capture the peptide-MHC-I complexes from C57BL/6 mouse samples. For human studies and for the generation of human aAPCs the W6/32 pan HLA-I antibody could prove to be a good candidate. Viral proteins that interact with the cytoplasmic portion of HLA-I could be also a viable option for affinity purification of peptide-MHC-I repertoire and generation of aAPCs.

In theory this technique can be applied to achieve both immunogenic and tolerogenic immune responses. AAPCs generated from normal cells or cells pulsed with self-antigen and combined with inhibitory signals and cytokines should generate antigen-specific regulatory $\mathrm{T}$ cell responses (prevent or treat autoimmune diseases). Along the same logic aAPCs could be generated to treat allergy or used as preventative vaccines to fight infectious diseases.

In summary, here we presented the scientific community with a concept that has the potential to revolutionize cancer vaccines and make it affordable to anybody in matter of days giving a chance also to the patients with very limited time on their hands. Of course, more research will be needed to perfect this technology, including but not limited to carrier's characteristics (material, size, shape, in vivo half-life, controlled release etc.), co-stimulatory molecules, cytokines and chemokines to use, route of delivery etc.

\section{Data availability}

Datasets are included with the manuscript.

Received: 19 June 2019; Accepted: 24 November 2019;

Published online: 12 December 2019

\section{References}

1. Merad, M., Sathe, P., Helft, J., Miller, J. \& Mortha, A. The dendritic cell lineage: ontogeny and function of dendritic cells and their subsets in the steady state and the inflamed setting. Annu. Rev. Immunol. 31, 563-604 (2013).

2. Fu, C. \& Jiang, A. Dendritic Cells and CD8 T Cell Immunity in Tumor Microenvironment. Frontiers in immunology, https://doi. org/10.3389/fimmu.2018.03059 (2018).

3. Palucka, K. \& Banchereau, J. Dendritic cell-based cancer therapeutic vaccines Karolina. Immunity, https://doi.org/10.1016/j. immuni.2013.07.004.Dendritic (2013).

4. Han, H. et al. A novel system of artificial antigen-presenting cells efficiently stimulates Flu peptide-specific cytotoxic T cells in vitro. Biochem. Biophys. Res. Commun., https://doi.org/10.1016/j.bbrc.2011.06.164 (2011).

5. Lu, X., Jiang, X., Liu, R., Zhao, H. \& Liang, Z. Adoptive transfer of pTRP2-specific CTLs expanding by bead-based artificial antigenpresenting cells mediates anti-melanoma response. Cancer Lett., https://doi.org/10.1016/i.canlet.2008.05.049 (2008).

6. Oelke, M. et al. Ex vivo induction and expansion of antigen-specific cytotoxic T cells by HLA-Ig-coated artificial antigen-presenting cells. Nat. Med., https://doi.org/10.1038/nm869 (2003).

7. Reddy, S. T., Rehor, A., Schmoekel, H. G., Hubbell, J. A. \& Swartz, M. A. In vivo targeting of dendritic cells in lymph nodes with poly(propylene sulfide) nanoparticles. J. Control. Release, https://doi.org/10.1016/j.jconrel.2006.01.006 (2006).

8. Shao, J. et al. Artificial antigen-presenting cells are superior to dendritic cells at inducing antigen-specific cytotoxic $\mathrm{T}$ lymphocytes. Cell. Immunol., https://doi.org/10.1016/j.cellimm.2018.10.002 (2018).

9. Steenblock, E. R. \& Fahmy, T. M. A comprehensive platform for ex vivo T-cell expansion based on biodegradable polymeric artificial antigen-presenting cells. Mol. Ther., https://doi.org/10.1038/mt.2008.11 (2008).

10. Ugel, S. et al. In vivo administration of artificial antigen-presenting cells activates low-avidity $\mathrm{T}$ cells for treatment of cancer. Cancer Res., https://doi.org/10.1158/0008-5472.CAN-09-0400 (2009).

11. Wang, C., Sun, W., Ye, Y., Bomba, H. N. \& Gu, Z. Bioengineering of artificial antigen presenting cells and lymphoid organs. Theranostics, https://doi.org/10.7150/thno.19017 (2017).

12. Laport, G. G. et al. Adoptive transfer of costimulated T cells induces lymphocytosis in patients with relapsed/refractory nonHodgkin lymphoma following CD34 +-selected hematopoietic cell transplantation. Blood, https://doi.org/10.1182/ blood-2003-01-0095 (2003).

13. Mitchell, M. S. et al. Phase I trial of adoptive immunotherapy with cytolytic T lymphocytes immunized against a tyrosinase epitope. J. Clin. Oncol., https://doi.org/10.1200/JCO.20.4.1075 (2002).

14. Igyártó, B. Z. et al. Skin-resident murine dendritic cell subsets promote distinct and opposing antigen-specific $\mathrm{T}$ helper cell responses. Immunity 35, 260-272 (2011).

15. Ebstein, F. et al. Proteasomes generate spliced epitopes by two different mechanisms and as efficiently as non-spliced epitopes. Sci. Rep. 6, 24032 (2016).

16. Hundal, J. et al. pVAC-Seq: A genome-guided in silico approach to identifying tumor neoantigens. Genome Med., https://doi. org/10.1186/s13073-016-0264-5 (2016).

17. Laumont, C. M. et al. Global proteogenomic analysis of human MHC class I-associated peptides derived from non-canonical reading frames. Nat. Commun., https://doi.org/10.1038/ncomms10238 (2016).

18. Liepe, J. et al. A large fraction of HLA class I ligands are proteasome-generated spliced peptides. Science (80-.), https://doi. org/10.1126/science.aaf4384 (2016).

19. Scheper, W. et al. Low and variable tumor reactivity of the intratumoral TCR repertoire in human cancers. Nature Medicine, https:// doi.org/10.1038/s41591-018-0266-5 (2019).

20. Bubeník, J. Tumour MHC class I downregulation and immunotherapy (Review). Oncology reports (2003). 


\section{Acknowledgements}

We are grateful to Dr. Jonathan Yewdell (NIAID) for providing the $\mathrm{H}-2 \mathrm{~Kb}$ plasmid and to Dr. Gerard Zurawski for the cetHS-puro plasmid. We also thank the animal facility and flow core for their help and support. The Baylor Scott \& White Health Foundation supported this work.

\section{Author contributions}

B.Z.I. conceptualized the one-step aAPC. Q.S. did all the experiments and analyzed the data. Q.S. and B.Z.I. designed experiments, interpreted the results and wrote the manuscript.

\section{Competing interests}

The authors declare no competing interests as defined by Nature Research, or other interests that might be perceived to influence the results and/or discussion reported in this paper. The authors have no competing financial and non-financial interests in relation to the work described. U.S. Provisional Patent Application $62 / 851,537$

\section{Additional information}

Correspondence and requests for materials should be addressed to B.Z.I.

Reprints and permissions information is available at www.nature.com/reprints.

Publisher's note Springer Nature remains neutral with regard to jurisdictional claims in published maps and institutional affiliations.

(c) (i) Open Access This article is licensed under a Creative Commons Attribution 4.0 International License, which permits use, sharing, adaptation, distribution and reproduction in any medium or format, as long as you give appropriate credit to the original author(s) and the source, provide a link to the Creative Commons license, and indicate if changes were made. The images or other third party material in this article are included in the article's Creative Commons license, unless indicated otherwise in a credit line to the material. If material is not included in the article's Creative Commons license and your intended use is not permitted by statutory regulation or exceeds the permitted use, you will need to obtain permission directly from the copyright holder. To view a copy of this license, visit http://creativecommons.org/licenses/by/4.0/.

(C) The Author(s) 2019 